\title{
Dirichlet problem for the Schrödinger operator on a cone
}

\author{
Lei Qiao ${ }^{1 *}$ and Guan-Tie Deng ${ }^{2}$
}

"Correspondence: qiaocqu@163.com

${ }^{1}$ Department of Mathematics and Information Science, Henan

University of Economics and Law, Zhengzhou 450002, P.R. China

Full list of author information is available at the end of the article

\begin{abstract}
In this article, a solution of the Dirichlet problem for the Schrödinger operator on a cone is constructed by the generalized Poisson integral with a slowly growing continuous boundary function. A solution of the Poisson integral for any continuous boundary function is also given explicitly by the Poisson integral with the generalized Poisson kernel depending on this boundary function.
\end{abstract}

MSC: 31B05; 31B10

Keywords: Dirichlet problem; stationary Schrödinger equation; cone

\section{Introduction and results}

Let $\mathbf{R}$ and $\mathbf{R}_{+}$be the set of all real numbers and the set of all positive real numbers respectively. We denote the $n$-dimensional Euclidean space by $\mathbf{R}^{n}(n \geq 2)$. A point in $\mathbf{R}^{n}$ is denoted by $P=\left(X, x_{n}\right)$, where $X=\left(x_{1}, x_{2}, \ldots, x_{n-1}\right)$. The Euclidean distance between two points $P$ and $Q$ in $\mathbf{R}^{n}$ is denoted by $|P-Q|$. Also $|P-O|$ with the origin $O$ of $\mathbf{R}^{n}$ is simply denoted by $|P|$. The boundary and the closure of a set $\mathbf{S}$ in $\mathbf{R}^{n}$ are denoted by $\partial \mathbf{S}$ and $\overline{\mathbf{S}}$ respectively.

We introduce a system of spherical coordinates $(r, \Theta), \Theta=\left(\theta_{1}, \theta_{2}, \ldots, \theta_{n-1}\right)$, in $\mathbf{R}^{n}$ which are related to Cartesian coordinates $\left(x_{1}, x_{2}, \ldots, x_{n-1}, x_{n}\right)$ by $x_{n}=r \cos \theta_{1}$.

The unit sphere and the upper half unit sphere in $\mathbf{R}^{n}$ are denoted by $\mathbf{S}^{n-1}$ and $\mathbf{S}_{+}^{n-1}$, respectively. For simplicity, a point $(1, \Theta)$ on $\mathbf{S}^{n-1}$ and the set $\{\Theta ;(1, \Theta) \in \Omega\}$ for a set $\Omega, \Omega \subset \mathbf{S}^{n-1}$, are often identified with $\Theta$ and $\Omega$, respectively. For two sets $\boldsymbol{\Xi} \subset \mathbf{R}_{+}$and $\Omega \subset \mathbf{S}^{n-1}$, the set $\left\{(r, \Theta) \in \mathbf{R}^{n} ; r \in \Xi,(1, \Theta) \in \Omega\right\}$ in $\mathbf{R}^{n}$ is simply denoted by $\Xi \times \Omega$.

For $P \in \mathbf{R}^{n}$ and $r>0$, let $B(P, r)$ denote an open ball with a center at $P$ and radius $r$ in $\mathbf{R}^{n} . S_{r}=\partial B(O, r)$. By $C_{n}(\Omega)$, we denote the set $\mathbf{R}_{+} \times \Omega$ in $\mathbf{R}^{n}$ with the domain $\Omega$ on $\mathbf{S}^{n-1}$. We call it a cone. We denote the sets $I \times \Omega$ and $I \times \partial \Omega$ with an interval on $\mathbf{R}$ by $C_{n}(\Omega ; I)$ and $S_{n}(\Omega ; I)$. By $S_{n}(\Omega ; r)$ we denote $C_{n}(\Omega) \cap S_{r}$. By $S_{n}(\Omega)$ we denote $S_{n}(\Omega ;(0,+\infty))$ which is $\partial C_{n}(\Omega)-\{O\}$. We denote the $(n-1)$-dimensional volume elements induced by the Euclidean metric on $S_{r}$ by $d S_{r}$.

Let $\mathcal{A}_{a}$ denote the class of nonnegative radial potentials $a(P)$, i.e., $0 \leq a(P)=a(r), P=$ $(r, \Theta) \in C_{n}(\Omega)$, such that $a \in L_{\text {loc }}^{b}\left(C_{n}(\Omega)\right)$ with some $b>n / 2$ if $n \geq 4$ and with $b=2$ if $n=2$ or $n=3$.

This article is devoted to the stationary Schrödinger equation

$$
\operatorname{Sch}_{a} u(P)=-\Delta u(P)+a(P) u(P)=0
$$

(0) 2012 Qiao and Deng; licensee Springer. This is an Open Access article distributed under the terms of the Creative Commons Attribution License (http://creativecommons.org/licenses/by/2.0), which permits unrestricted use, distribution, and reproduction in any medium, provided the original work is properly cited. 
where $P \in C_{n}(\Omega), \Delta$ is the Laplace operator and $a \in \mathcal{A}_{a}$. These solutions called $a$-harmonic functions or generalized harmonic functions are associated with the operator $\mathrm{Sch}_{a}$. Note that they are (classical) harmonic functions in the case $a=0$. Under these assumptions, the operator $\operatorname{Sch}_{a}$ can be extended in the usual way from the space $C_{0}^{\infty}\left(C_{n}(\Omega)\right)$ to an essentially self-adjoint operator on $L^{2}\left(C_{n}(\Omega)\right)$ (see [1-3]). We will denote it $\operatorname{Sch}_{a}$ as well. This last one has a Green's function $G(\Omega, a)(P, Q)$. Here $G(\Omega, a)(P, Q)$ is positive on $C_{n}(\Omega)$ and its inner normal derivative $\partial G(\Omega, a)(P, Q) / \partial n_{Q} \geq 0$. We denote this derivative by $\mathbb{P}(\Omega, a)(P, Q)$, which is called the Poisson $a$-kernel with respect to $C_{n}(\Omega)$. We remark that $G(\Omega, 0)(P, Q)$ and $\mathbb{P}(\Omega, 0)(P, Q)$ are the Green's function and Poisson kernel of the Laplacian in $C_{n}(\Omega)$ respectively.

Given a domain $D \subset \mathbf{R}^{n}$ and a continuous function $u$ on $\partial(D)$, we say that $h$ is a solution of the Dirichlet problem for the Schrödinger operator on $D$ with $u$ if $\operatorname{Sch}_{a} h=0$ in $D$ and

$$
\lim _{P \in D, P \rightarrow Q} h(P)=u(Q)
$$

for every $Q \in \partial(D)$. Note that $h$ is a solution of the classical Dirichlet problem for the Laplacian in the case $a=0$.

Let $\Delta^{*}$ be a Laplace-Beltrami operator (the spherical part of the Laplace) on $\Omega \subset \mathbf{S}^{n-1}$ and $\lambda_{j}\left(j=1,2,3, \ldots, 0<\lambda_{1}<\lambda_{2} \leq \lambda_{3} \leq \ldots\right)$ be the eigenvalues of the eigenvalue problem for $\Delta^{*}$ on $\Omega$ (see, e.g., [4, p. 41])

$$
\begin{aligned}
& \Delta^{\prime \prime} \varphi(\Theta)+\lambda \varphi(\Theta)=0 \quad \text { in } \Omega, \\
& \varphi(\Theta)=0 \quad \text { on } \partial \Omega .
\end{aligned}
$$

Corresponding eigenfunctions are denoted by $\varphi_{j v}\left(1 \leq v \leq v_{j}\right)$, where $v_{j}$ is the multiplicity of $\lambda_{j}$. We set $\lambda_{0}=0$, norm the eigenfunctions in $L^{2}(\Omega)$ and $\varphi_{1}=\varphi_{11}>0$. Then there exist two positive constants $d_{1}$ and $d_{2}$ such that

$$
d_{1} \delta(P) \leq \varphi_{1}(\Theta) \leq d_{2} \delta(P)
$$

for $P=(1, \Theta) \in \Omega$ (see Courant and Hilbert [5]), where $\delta(P)=\inf _{Q \in \partial C_{n}(\Omega)}|P-Q|$.

In order to ensure the existences of $\lambda_{j}(j=1,2,3, \ldots)$. We put a rather strong assumption on $\Omega$ : if $n \geq 3$, then $\Omega$ is a $C^{2, \alpha}$-domain $(0<\alpha<1)$ on $\mathbf{S}^{n-1}$ surrounded by a finite number of mutually disjoint closed hypersurfaces (e.g., see [6, pp. 88-89] for the definition of $C^{2, \alpha}$-domain). Then $\varphi_{j v} \in C^{2}(\bar{\Omega})\left(j=1,2,3, \ldots, 1 \leq v \leq v_{j}\right)$ and $\partial \varphi_{1} / \partial n>0$ on $\partial \Omega$ (here and below, $\partial / \partial n$ denotes differentiation along the interior normal).

Hence well-known estimates (see, e.g., [7, p. 14]) imply the following inequality:

$$
\sum_{v=1}^{v_{j}} \varphi_{j v}(\Theta) \frac{\partial \varphi_{j v}(\Phi)}{\partial n_{\Phi}} \leq M(n) j^{2 n-1}
$$

where the symbol $M(n)$ denotes a constant depending only on $n$.

Let $V_{j}(r)$ and $W_{j}(r)$ stand, respectively, for the increasing and nonincreasing, as $r \rightarrow+\infty$, solutions of the equation

$$
-Q^{\prime \prime}(r)-\frac{n-1}{r} Q^{\prime}(r)+\left(\frac{\lambda_{j}}{r^{2}}+a(r)\right) Q(r)=0, \quad 0<r<\infty,
$$


normalized under the condition $V_{j}(1)=W_{j}(1)=1$.

We shall also consider the class $\mathscr{B}_{a}$, consisting of the potentials $a \in \mathcal{A}_{a}$ such that there exists a finite $\operatorname{limit}_{\lim } \rightarrow \infty r^{2} a(r)=k \in[0, \infty)$; moreover, $r^{-1}\left|r^{2} a(r)-k\right| \in L(1, \infty)$. If $a \in$ $\mathscr{B}_{a}$, then the solutions of Equation (1.1) are continuous (see [8]).

In the rest of the article, we assume that $a \in \mathscr{B}_{a}$ and we shall suppress this assumption for simplicity. Further, we use the standard notations $u^{+}=\max (u, 0), u^{-}=-\min (u, 0),[d]$ is the integer part of $d$ and $d=[d]+\{d\}$, where $d$ is a positive real number.

Denote

$$
\iota_{j, k}^{ \pm}=\frac{2-n \pm \sqrt{(n-2)^{2}+4\left(k+\lambda_{j}\right)}}{2} \quad(j=0,1,2,3, \ldots) .
$$

It is known (see [9]) that in the case under consideration the solutions to Equation (1.4) have the asymptotics

$$
V_{j}(r) \sim d_{3} r^{r_{j}^{+}, k}, \quad W_{j}(r) \sim d_{4} r^{l_{j}^{-}, k}, \quad \text { as } r \rightarrow \infty,
$$

where $d_{3}$ and $d_{4}$ are some positive constants.

If $a \in \mathcal{A}_{a}$, it is known that the following expansion for the Green function $G(\Omega, a)(P, Q)$ (see [10, Ch. 11], [1, 11])

$$
G(\Omega, a)(P, Q)=\sum_{j=0}^{\infty} \frac{1}{\chi^{\prime}(1)} V_{j}(\min (r, t)) W_{j}(\max (r, t))\left(\sum_{\nu=1}^{v_{j}} \varphi_{j \nu}(\Theta) \varphi_{j v}(\Phi)\right),
$$

where $P=(r, \Theta), Q=(t, \Phi), r \neq t$ and $\chi^{\prime}(s)=\left.w\left(W_{1}(r), V_{1}(r)\right)\right|_{r=s}$, is their Wronskian. The series converges uniformly if either $r \leq s t$ or $t \leq s r(0<s<1)$.

For a nonnegative integer $m$ and two points $P=(r, \Theta), Q=(t, \Phi) \in C_{n}(\Omega)$, we put

$$
K(\Omega, a, m)(P, Q)= \begin{cases}0 & \text { if } 0<t<1, \\ \widetilde{K}(\Omega, a, m)(P, Q) & \text { if } 1 \leq t<\infty,\end{cases}
$$

where

$$
\widetilde{K}(\Omega, a, m)(P, Q)=\sum_{j=0}^{m} \frac{1}{\chi^{\prime}(1)} V_{j}(r) W_{j}(t)\left(\sum_{v=1}^{v_{j}} \varphi_{j v}(\Theta) \varphi_{j v}(\Phi)\right) .
$$

We introduce another function of $P=(r, \Theta) \in C_{n}(\Omega)$ and $Q=(t, \Phi) \in C_{n}(\Omega)$

$$
G(\Omega, a, m)(P, Q)=G(\Omega, a)(P, Q)-K(\Omega, a, m)(P, Q) .
$$

The generalized Poisson kernel $\mathbb{P}(\Omega, a, m)(P, Q)\left(P=(r, \Theta) \in C_{n}(\Omega), Q=(t, \Phi) \in S_{n}(\Omega)\right)$ with respect to $C_{n}(\Omega)$ is defined by

$$
\mathbb{P}(\Omega, a, m)(P, Q)=\frac{\partial G(\Omega, a, m)(P, Q)}{\partial n_{Q}} .
$$

In fact,

$$
\mathbb{P}(\Omega, a, 0)(P, Q)=\mathbb{P}(\Omega, a)(P, Q) .
$$


We remark that the kernel function $\mathbb{P}(\Omega, 0, m)(P, Q)$ coincides with the one in Yoshida and Miyamoto [12] (see [10, Ch. 11]).

Put

$$
U(\Omega, a, m ; u)(P)=\int_{S_{n}(\Omega)} \mathbb{P}(\Omega, a, m)(P, Q) u(Q) d \sigma_{Q},
$$

where $u(Q)$ is a continuous function on $\partial C_{n}(\Omega)$ and $d \sigma_{Q}$ is a surface area element on $S_{n}(\Omega)$.

With regard to classical solutions of the Dirichlet problem for the Laplacian, Yoshida and Miyamoto [12, Theorem 1] proved the following result.

Theorem A If $u$ is a continuous function on $\partial C_{n}(\Omega)$ satisfying

$$
\int_{S_{n}(\Omega)} \frac{|u(t, \Phi)|}{1+t^{t^{+}+1,0}+n-1} d \sigma_{Q}<\infty
$$

then $U(\Omega, 0, m ; u)(P)$ is a classical solution of the Dirichlet problem on $C_{n}(\Omega)$ with $g$ and satisfies

$$
\lim _{r \rightarrow \infty, P=(r, \Theta) \in C_{n}(\Omega)} r^{-l_{m+1,0}^{+}} U(\Omega, 0, m ; u)(P)=0 .
$$

Our first aim is to give growth properties at infinity for $U(\Omega, a, m ; u)(P)$.

Theorem 1 Let $\gamma \geq 0$ (resp. $\gamma<0), \iota_{[\gamma], k}^{+}+\{\gamma\}>-\iota_{1, k}^{+}+1$ (resp. $\left.-\iota_{[-\gamma], k}^{+}-\{-\gamma\}>-\iota_{1, k}^{+}+1\right)$ and

$$
\begin{aligned}
& \iota_{[\gamma], k}^{+}+\{\gamma\}-n+1 \leq \iota_{m+1, k}^{+}<\iota_{[\gamma], k}^{+}+\{\gamma\}-n+2 \\
& \left(\text { resp.s }-\iota_{[-\gamma], k}^{+}-\{-\gamma\}-n+1 \leq \iota_{m+1, k}^{+}<-\iota_{[-\gamma], k}^{+}-\{-\gamma\}-n+2\right) .
\end{aligned}
$$

If $u$ is a measurable function on $\partial C_{n}(\Omega)$ satisfying

$$
\left.\int_{S_{n}(\Omega)} \frac{|u(t, \Phi)|}{1+t^{t^{+}}[\gamma, k+\{\gamma\}} d \sigma_{Q}<\infty \quad\left(\text { resp. } \int_{S_{n}(\Omega)}|u(t, \Phi)|\left(1+t^{\left.t^{+}-\gamma\right], k}+\{-\gamma\}\right]\right) d \sigma_{Q}<\infty\right),
$$

then

$$
\begin{aligned}
& \lim _{r \rightarrow \infty, P=(r, \Theta) \in C_{n}(\Omega)} r^{-\iota_{[\gamma], k}^{+}-\{\gamma\}+n-1} U(\Omega, a, m ; u)(P)=0
\end{aligned}
$$

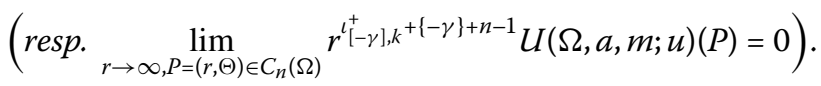

Next, we are concerned with solutions of the Dirichlet problem for the Schrödinger operator on $C_{n}(\Omega)$.

Theorem 2 Let $\gamma$ and $\iota_{m+1, k}^{+}$be as in Theorem 1. If $u$ is a continuous function on $\partial C_{n}(\Omega)$ satisfying (1.6), then $U(\Omega, a, m ; u)(P)$ is a solution of the Dirichlet problem for the Schrödinger operator on $C_{n}(\Omega)$ with $u$ and (1.7) (resp. (1.8)) holds.

If we take $\iota_{[\gamma], k}^{+}+\{\gamma\}=\iota_{m+1, k}^{+}+n-1$, then we immediately have the following corollary, which is just Theorem A in the case $a=0$. 
Corollary If $u$ is a continuous function on $\partial C_{n}(\Omega)$ satisfying

$$
\int_{S_{n}(\Omega)} \frac{|u(t, \Phi)|}{1+t^{\iota^{+}+1, k}+n-1} d \sigma_{Q}<\infty
$$

then $U(\Omega, a, m ; u)(P)$ is a solution of the Dirichlet problem for the Schrödinger operator on $C_{n}(\Omega)$ with $u$ and satisfies

$$
\lim _{r \rightarrow \infty, P=(r, \Theta) \in C_{n}(\Omega)} r^{-\iota_{m+1, k}^{+}} U(\Omega, a, m ; u)(P)=0 .
$$

By using Corollary, we can give a solution of the Dirichlet problem for any continuous function on $\partial C_{n}(\Omega)$.

Theorem 3 If $u$ is a continuous function on $\partial C_{n}(\Omega)$ satisfying $(1.9)$ and $h(r, \Theta)$ is a solution of the Dirichlet problem for the Schrödinger operator on $C_{n}(\Omega)$ with u satisfying

$$
\lim _{r \rightarrow \infty, P=(r, \Theta) \in C_{n}(\Omega)} r^{-\iota_{m+1, k}^{+}} h^{+}(P)=0,
$$

then

$$
h(P)=U(\Omega, a, m ; u)(P)+\sum_{j=0}^{m}\left(\sum_{v=1}^{v_{j}} d_{j v} \varphi_{j v}(\Theta)\right) V_{j}(r),
$$

where $P=(r, \Theta) \in C_{n}(\Omega)$ and $d_{j v}$ are constants.

\section{Lemmas}

Throughout this article, let $M$ denote various constants independent of the variables in questions, which may be different from line to line.

\section{Lemma 1}

$$
\begin{aligned}
& |\mathbb{P}(\Omega, a)(P, Q)| \leq M r^{\iota_{1}^{-}} t^{t_{1, k}^{+}-1} \\
& \left(\operatorname{resp} .|\mathbb{P}(\Omega, a)(P, Q)| \leq M r^{r_{1}^{+}, k} t^{t_{1, k}^{-}}{ }^{-1}\right)
\end{aligned}
$$

for any $P=(r, \Theta) \in C_{n}(\Omega)$ and any $Q=(t, \Phi) \in S_{n}(\Omega)$ satisfying $0<\frac{t}{r} \leq \frac{4}{5}$ (resp. $\left.0<\frac{r}{t} \leq \frac{4}{5}\right)$;

$$
|\mathbb{P}(\Omega, 0)(P, Q)| \leq M \frac{1}{t^{n-1}}+M \frac{r}{|P-Q|^{n}}
$$

for any $P=(r, \Theta) \in C_{n}(\Omega)$ and any $Q=(t, \Phi) \in S_{n}\left(\Omega ;\left(\frac{4}{5} r, \frac{5}{4} r\right)\right)$.

Proof (2.1) and (2.2) are obtained by Kheyfits (see [10, Ch. 11]). (2.3) follows from Azarin (see [13, Lemma 4 and Remark]).

Lemma 2 (see [1]) For a nonnegative integer $m$, we have

$$
|\mathbb{P}(\Omega, a, m)(P, Q)| \leq M(n, m, s) V_{m+1}(r) \frac{W_{m+1}(t)}{t} \varphi_{1}(\Theta) \frac{\partial \varphi_{1}(\Phi)}{\partial n_{\Phi}}
$$


for any $P=(r, \Theta) \in C_{n}(\Omega)$ and $Q=(t, \Phi) \in S_{n}(\Omega)$ satisfying $r \leq$ st $(0<s<1)$, where $M(n, m, s)$ is a constant dependent of $n, m$ and $s$.

Lemma 3 (see [2, Theorem 1]) If $u(r, \Theta)$ is a solution of Equation (1.1) on $C_{n}(\Omega)$ satisfying

$$
\int_{\Omega} u^{+}(r, \Theta) d S_{1}=O\left(r^{i^{+}, k}\right), \quad \text { as } r \rightarrow \infty
$$

then

$$
u(r, \Theta)=\sum_{j=0}^{m}\left(\sum_{v=1}^{v_{j}} d_{j v} \varphi_{j v}(\Theta)\right) V_{j}(r) .
$$

Lemma 4 Obviously, the conclusion of Lemma 3 holds true if (2.5) is replaced by

$$
\lim _{r \rightarrow \infty,(r, \Theta) \in C_{n}(\Omega)} r^{-\iota_{m+1, k}^{+}} u^{+}(r, \Theta)=0 .
$$

Proof Since

$$
V_{m+1}(r) \sim r^{\iota^{+}+1, k} \quad \text { as } r \rightarrow \infty
$$

from (1.5) and

$$
\iota_{m+1, k}^{+} \geq \iota_{m, k}^{+}
$$

(2.6) gives that (2.5) holds, from which the conclusion immediately follows.

\section{Proof of Theorem 1}

We only prove the case $\gamma \geq 0$, the remaining case $\gamma<0$ can be proved similarly.

For any $\epsilon>0$, there exists $R_{\epsilon}>1$ such that

$$
\int_{S_{n}\left(\Omega ;\left(R_{\epsilon}, \infty\right)\right)} \frac{|u(Q)|}{\left.1+t^{t^{+}} \mid \gamma\right], k+\{\gamma\}} d \sigma_{Q}<\epsilon
$$

The relation $G(\Omega, a)(P, Q) \leq G(\Omega, 0)(P, Q)$ implies this inequality (see [14])

$$
\mathbb{P}(\Omega, a)(P, Q) \leq \mathbb{P}(\Omega, 0)(P, Q) .
$$

For $0<s<\frac{4}{5}$ and any fixed point $P=(r, \Theta) \in C_{n}(\Omega)$ satisfying $r>\frac{5}{4} R_{\epsilon}$, let $I_{1}=$ $S_{n}(\Omega ;(0,1)), I_{2}=S_{n}\left(\Omega ;\left[1, R_{\epsilon}\right]\right), I_{3}=S_{n}\left(\Omega ;\left(R_{\epsilon}, \frac{4}{5} r\right]\right), I_{4}=S_{n}\left(\Omega ;\left(\frac{4}{5} r, \frac{5}{4} r\right)\right), I_{5}=S_{n}\left(\Omega ;\left[\frac{5}{4} r, \frac{r}{s}\right)\right)$, $I_{6}=S_{n}\left(\Omega ;\left[1, \frac{r}{s}\right)\right)$ and $I_{7}=S_{n}\left(\Omega ;\left[\frac{r}{s}, \infty\right)\right)$, we write

$$
U(\Omega, a, m ; u)(P) \leq \sum_{i=1}^{7} U_{\Omega, a, i}(P),
$$

where

$$
U_{\Omega, a, i}(P)=\int_{I_{i}}|\mathbb{P}(\Omega, a)(P, Q)||u(Q)| d \sigma_{Q} \quad(i=1,2,3,4,5),
$$




$$
\begin{aligned}
& U_{\Omega, a, 6}(P)=\int_{I_{6}}|\mathbb{P}(\Omega, a, m)(P, Q)||u(Q)| d \sigma_{Q}, \\
& U_{\Omega, a, 7}(P)=\int_{I_{7}}\left|\frac{\partial \widetilde{K}(\Omega, a, m)(P, Q)}{\partial n_{Q}}\right||u(Q)| d \sigma_{Q} .
\end{aligned}
$$

By $\iota_{[\gamma], k}^{+}+\{\gamma\}>-\iota_{1, k}^{+}+1,(1.6),(2.1)$ and (3.1), we have the following growth estimates

$$
\begin{aligned}
U_{\Omega, a, 2}(P) & \leq M r^{l_{1, k}^{-}} \int_{I_{2}} t^{l_{1, k}^{+}-1}|u(Q)| d \sigma_{Q} \\
& \leq M r^{l_{1, k}^{-}} R_{\epsilon}^{l_{+}^{+}[\gamma], k}+\{\gamma\}+l_{1, k}^{+}-1 \\
U_{\Omega, a, 1}(P) & \leq M r^{l_{1, k}^{-}}, \\
U_{\Omega, a, 3}(P) & \leq M \epsilon r^{l_{[\gamma], k}^{+}},
\end{aligned}
$$

We obtain by $\iota_{m+1, k}^{+} \geq \iota_{[\gamma], k}^{+}+\{\gamma\}-n+1,(2.2)$ and (3.1)

$$
\begin{aligned}
& U_{\Omega, a, 5}(P) \leq M r^{l_{1, k}^{+}} \int_{S_{n}(\Omega ;[(5 / 4) r, \infty))} t^{l_{1, k}^{-}-1}|u(Q)| d \sigma_{Q} \\
& \leq M r^{l_{1, k}^{+}} \int_{S_{n}(\Omega ;[(5 / 4) r, \infty))} t^{l_{[\gamma], k}^{+}+\{\gamma\}+l_{1, k}^{-}-1} \frac{|u(Q)|}{t^{t_{[\gamma], k}^{+}+\{\gamma\}}} d \sigma_{Q} \\
& \leq M \in r^{l^{+}[\gamma], k}+\{\gamma\}-n+1 .
\end{aligned}
$$

By (2.3) and (3.2), we consider the inequality

$$
U_{\Omega, a, 4}(P) \leq U_{\Omega, 0,4}(P) \leq U_{\Omega, 0,4}^{\prime}(P)+U_{\Omega, 0,4}^{\prime \prime}(P)
$$

where

$$
U_{\Omega, 0,4}^{\prime}(P)=M \int_{I_{4}} t^{1-n}|u(Q)| d \sigma_{Q}, \quad U_{\Omega, 0,4}^{\prime \prime}(P)=M r \int_{I_{4}} \frac{|u(Q)|}{|P-Q|^{n}} d \sigma_{Q}
$$

We first have

$$
\begin{aligned}
U_{\Omega, 0,4}^{\prime}(P) & =M \int_{I_{4}} t^{l_{1, k}^{+}+l_{1, k}^{-}-1}|u(Q)| d \sigma_{Q} \\
& \leq M r^{l_{1, k}^{+}} \int_{S_{n}(\Omega ;((4 / 5) r, \infty))} t^{l_{1, k}^{-}-1}|u(Q)| d \sigma_{Q} \\
& \leq M \epsilon r^{\left.l^{+} \gamma\right], k+\{\gamma\}-n+1},
\end{aligned}
$$

which is similar to the estimate of $U_{\Omega, a, 5}(P)$.

Next, we shall estimate $U_{\Omega, 0,4}^{\prime \prime}(P)$. Take a sufficiently small positive number $d_{5}$ such that $I_{4} \subset B\left(P, \frac{1}{2} r\right)$ for any $P=(r, \Theta) \in \Pi\left(d_{5}\right)$, where

$$
\Pi\left(d_{5}\right)=\left\{P=(r, \Theta) \in C_{n}(\Omega) ; \inf _{z \in \partial \Omega}|(1, \Theta)-(1, z)|<d_{5}, 0<r<\infty\right\}
$$

and divide $C_{n}(\Omega)$ into two sets $\Pi\left(d_{5}\right)$ and $C_{n}(\Omega)-\Pi\left(d_{5}\right)$. 
If $P=(r, \Theta) \in C_{n}(\Omega)-\Pi\left(d_{5}\right)$, then there exists a positive $d_{5}^{\prime}$ such that $|P-Q| \geq d_{5}^{\prime} r$ for any $Q \in S_{n}(\Omega)$, and hence

$$
\begin{aligned}
U_{\Omega, 0,4}^{\prime \prime}(P) & \leq M \int_{I_{4}} t^{1-n}|u(Q)| d \sigma_{Q} \\
& \leq M \epsilon r^{\left.l^{+} \gamma\right], k}+\{\gamma\}-n+1,
\end{aligned}
$$

which is similar to the estimate of $U_{\Omega, 0,4}^{\prime}(P)$.

We shall consider the case $P=(r, \Theta) \in \Pi\left(d_{5}\right)$. Now put

$$
H_{i}(P)=\left\{Q \in I_{4} ; 2^{i-1} \delta(P) \leq|P-Q|<2^{i} \delta(P)\right\}
$$

Since $S_{n}(\Omega) \cap\left\{Q \in \mathbf{R}^{n}:|P-Q|<\delta(P)\right\}=\varnothing$, we have

$$
U_{\Omega, 0,4}^{\prime \prime}(P)=M \sum_{i=1}^{i(P)} \int_{H_{i}(P)} r \frac{|u(Q)|}{|P-Q|^{n}} d \sigma_{Q},
$$

where $i(P)$ is a positive integer satisfying $2^{i(P)-1} \delta(P) \leq \frac{r}{2}<2^{i(P)} \delta(P)$.

Since we see from (1.2)

$$
r \varphi_{1}(\Theta) \leq M \delta(P)
$$

for $P=(r, \Theta) \in C_{n}(\Omega)$. Similar to the estimate of $U_{\Omega, 0,4}^{\prime}(P)$, we obtain

$$
\begin{aligned}
& \int_{H_{i}(P)} r \frac{|u(Q)|}{|P-Q|^{n}} d \sigma_{Q} \\
& \quad \leq \int_{H_{i}(P)} r \frac{|u(Q)|}{\left(2^{i-1} \delta(P)\right)^{n}} d \sigma_{Q} \\
& \leq M 2^{(1-i) n} \int_{H_{i}(P)} t^{1-n}|u(Q)| d \sigma_{Q} \\
& \leq M \epsilon r^{l^{+}[\gamma], k}+\{\gamma\}-n+1
\end{aligned}
$$

for $i=0,1,2, \ldots, i(P)$.

So

$$
U_{\Omega, 0,4}^{\prime \prime}(P) \leq M \in r^{l^{+}[\gamma], k^{+}\{\gamma\}-n+1} .
$$

We only consider $U_{\Omega, a, 6}(P)$ in the case $m \geq 1$, since $U_{\Omega, a, 6}(P) \equiv 0$ for $m=0$. By the definition of $\widetilde{K}(\Omega, a, m),(1.3)$ and Lemma 2 , we see

$$
U_{\Omega, a, 6}(P) \leq \frac{M}{\chi^{\prime}(1)} \sum_{j=0}^{m} j^{2 n-1} q_{j}(r),
$$

where

$$
q_{j}(r)=V_{j}(r) \int_{I_{6}} \frac{W_{j}(t)|u(Q)|}{t} d \sigma_{Q} .
$$


To estimate $q_{j}(r)$, we write

$$
q_{j}(r) \leq q_{j}^{\prime}(r)+q_{j}^{\prime \prime}(r)
$$

where

$$
q_{j}^{\prime}(r)=V_{j}(r) \int_{I_{2}} \frac{W_{j}(t)|u(Q)|}{t} d \sigma_{Q}, \quad q_{j}^{\prime \prime}(r)=V_{j}(r) \int_{S_{n}\left(\Omega ;\left(R_{\epsilon}, r / s\right)\right)} \frac{W_{j}(t)|u(Q)|}{t} d \sigma_{Q} .
$$

Notice that

$$
V_{j}(r) \frac{V_{m+1}(t)}{V_{j}(t) t} \leq M \frac{V_{m+1}(r)}{r} \leq M r^{i^{+}+1, k}-1 \quad\left(t \geq 1, R_{\epsilon}<\frac{r}{s}\right)
$$

Thus, by $\iota_{m+1, k}^{+}<\iota_{[\gamma], k}^{+}+\{\gamma\}-n+2$, (1.5) and (1.6) we conclude

$$
\begin{aligned}
q_{j}^{\prime}(r) & =V_{j}(r) \int_{I_{2}} \frac{|u(Q)|}{V_{j}(t) t^{n-1}} d \sigma_{Q} \\
& \leq M V_{j}(r) \int_{I_{2}} \frac{V_{m+1}(t)}{t^{\iota^{+}+1, k}} \frac{|u(Q)|}{V_{j}(t) t^{n-1}} d \sigma_{Q} \\
& \leq M r^{\iota_{m+1, k}^{+}}-1 R_{\epsilon}^{\iota_{[+}^{+}, k+\{\gamma\}-\iota_{m+1, k^{+}}^{-n+2}} .
\end{aligned}
$$

Analogous to the estimate of $q_{j}^{\prime}(r)$, we have

$$
q_{j}^{\prime \prime}(r) \leq M \epsilon r^{l^{+}}[\gamma], k+\{\gamma\}-n+1
$$

Thus we can conclude that

$$
q_{j}(r) \leq M \in r^{l^{+}}[\gamma], k+\{\gamma\}-n+1,
$$

which yields

$$
U_{\Omega, a, 6}(P) \leq M \in r^{l^{+}[\gamma], k^{+}+\{\gamma\}-n+1} .
$$

By $\iota_{m+1, k}^{+} \geq \iota_{[\gamma], k}^{+}+\{\gamma\}-n+1,(1.5),(2.4)$ and (3.1) we have

$$
\begin{aligned}
U_{\Omega, 0,7}(P) & \leq M V_{m+1}(r) \int_{I_{7}} \frac{|u(Q)|}{V_{m+1}(t) t^{n-1}} d \sigma_{Q} \\
& \leq M \epsilon r^{l^{+}[\gamma], k^{+}\{\gamma\}-n+1} .
\end{aligned}
$$

Combining (3.3)-(3.11), we obtain that if $R_{\epsilon}$ is sufficiently large and $\epsilon$ is sufficiently small, then $U(\Omega, a, m ; u)(P)=o\left(r^{\left.l^{+} \gamma\right], k^{+}\{\gamma\}-n+1}\right)$ as $r \rightarrow \infty$, where $P=(r, \Theta) \in C_{n}(\Omega)$. Then we complete the proof of Theorem 1 . 


\section{Proof of Theorem 2}

For any fixed $P=(r, \Theta) \in C_{n}(\Omega)$, take a number satisfying $R>\max \left(1, \frac{r}{s}\right)\left(0<s<\frac{4}{5}\right)$. By $\iota_{m+1, k}^{+} \geq \iota_{[\gamma], k}^{+}+\{\gamma\}-n+1,(1.4),(1.6)$ and (2.4), we have

$$
\begin{aligned}
& \int_{S_{n}(\Omega ;(R, \infty))}|\mathbb{P}(\Omega, a, m)(P, Q)||u(Q)| d \sigma_{Q} \\
& \leq M V_{m+1}(r) \varphi_{1}(\Theta) \int_{S_{n}(\Omega ;(R, \infty))} \frac{|u(Q)|}{t^{\iota^{+}+1, k}+n-1} d \sigma_{Q} \\
& \leq M r^{\iota^{+}+1, k} \varphi_{1}(\Theta) \int_{S_{n}(\Omega ;(r / s, \infty))} t^{t^{+}[\gamma], k+\{\gamma\}-l_{m+1, k}^{+}-n+1} \frac{|u(Q)|}{t^{t^{+}}[\gamma], k^{+}+\{\gamma\}} d \sigma_{Q} \\
& \leq M r^{\iota^{+}[\gamma], k^{+}+\{\gamma\}-n+1} \varphi_{1}(\Theta) \int_{\left.S_{n}(\Omega ;(r / s, \infty))\right)} \frac{|u(Q)|}{t^{t^{+}[\gamma], k^{+}+\{\gamma\}}} d \sigma_{Q} \\
& \leq M r^{\left.l^{+} \gamma\right], k^{+}\{\gamma\}-n+1} \varphi_{1}(\Theta) \\
& <\infty \text {. }
\end{aligned}
$$

Thus $U(\Omega, a, m ; u)(P)$ is finite for any $P \in C_{n}(\Omega)$. Since $\mathbb{P}(\Omega, a, m)(P, Q)$ is a generalized harmonic function of $P \in C_{n}(\Omega)$ for any fixed $Q \in S_{n}(\Omega), U(\Omega, a, m ; u)(P)$ is also a generalized harmonic function of $P \in C_{n}(\Omega)$. That is to say, $U(\Omega, a, m ; u)(P)$ is a solution of Equation (1.1) on $C_{n}(\Omega)$.

Now we study the boundary behavior of $U(\Omega, a, m ; u)(P)$. Let $Q^{\prime}=\left(t^{\prime}, \Phi^{\prime}\right) \in \partial C_{n}(\Omega)$ be any fixed point and $l$ be any positive number satisfying $l>\max \left(t^{\prime}+1, \frac{4}{5} R\right)$.

Set $\chi_{S(l)}$ is a characteristic function of $S(l)=\left\{Q=(t, \Phi) \in \partial C_{n}(\Omega), t \leq l\right\}$ and write

$$
U(\Omega, a, m ; u)(P)=U^{\prime}(P)-U^{\prime \prime}(P)+U^{\prime \prime \prime}(P),
$$

where

$$
\begin{aligned}
& U^{\prime}(P)=\int_{S_{n}(\Omega ;(0,(5 / 4) l])} \mathbb{P}(\Omega, a)(P, Q) u(Q) d \sigma_{Q}, \\
& U^{\prime \prime}(P)=\int_{S_{n}(\Omega ;(1,(5 / 4) l])} \frac{\partial K(\Omega, a, m)(P, Q)}{\partial n_{Q}} u(Q) d \sigma_{Q}, \\
& U^{\prime \prime \prime}(P)=\int_{S_{n}(\Omega ;((5 / 4) l, \infty))} \mathbb{P}(\Omega, a, m)(P, Q) u(Q) d \sigma_{Q} .
\end{aligned}
$$

Notice that $U^{\prime}(P)$ is the Poisson $a$-integral of $u(Q) \chi_{S(5 / 4) l)}$, we have $\lim _{P \rightarrow Q^{\prime}, P \in C_{n}(\Omega)} U^{\prime}(P)=u\left(Q^{\prime}\right)$. Since $\lim _{\Theta \rightarrow \Phi^{\prime}} \varphi_{j v}(\Theta)=0\left(j=1,2,3, \ldots ; 1 \leq v \leq v_{j}\right)$ as $P=$ $(r, \Theta) \rightarrow Q^{\prime}=\left(t^{\prime}, \Phi^{\prime}\right) \in S_{n}(\Omega)$, we have $\lim _{P \rightarrow Q^{\prime}, P \in C_{n}(\Omega)} U^{\prime \prime}(P)=0$ from the definition of

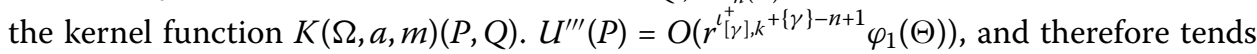
to zero.

So the function $U(\Omega, a, m ; u)(P)$ can be continuously extended to $\overline{C_{n}(\Omega)}$ such that

$$
\lim _{P \rightarrow Q^{\prime}, P \in C_{n}(\Omega)} U(\Omega, a, m ; u)(P)=u\left(Q^{\prime}\right)
$$

for any $Q^{\prime}=\left(t^{\prime}, \Phi^{\prime}\right) \in \partial C_{n}(\Omega)$ from the arbitrariness of $l$. Thus we complete the proof of Theorem 2 from Theorem 1 . 


\section{Proof of Theorem 3}

From Corollary, we have the solution $U(\Omega, a, m ; u)(P)$ of the Dirichlet problem on $C_{n}(\Omega)$ with $u$ satisfying (1.9). Consider the function $h(P)-U(\Omega, a, m ; u)(P)$. Then it follows that this is the solution of Equation (1.1) in $C_{n}(\Omega)$ and vanishes continuously on $\partial C_{n}(\Omega)$.

Since

$$
0 \leq(h-U(\Omega, a, m ; u))^{+}(P) \leq h^{+}(P)+(U(\Omega, a, m ; u))^{-}(P)
$$

for any $P \in C_{n}(\Omega)$, we have

$$
\lim _{r \rightarrow \infty, P=(r, \Theta) \in C_{n}(\Omega)} r^{-\iota_{m+1, k}^{+}}(h-U(\Omega, a, m ; u))^{+}(P)=0
$$

from (1.10) and (1.11). Then the conclusions of Theorem 3 follow immediately from Lemma 4.

\section{Competing interests}

The authors declare that they have no competing interests.

\section{Authors' contributions}

The authors declare that the study was realized in collaboration with the same responsibility. All authors read and approved the final manuscript.

\section{Author details}

'Department of Mathematics and Information Science, Henan University of Economics and Law, Zhengzhou 450002, P.R. China. ${ }^{2}$ School of Mathematical Science, Laboratory of Mathematics and Complex Systems, MOE Beijing Normal University, Beijing 100875, P.R. China.

\section{Acknowledgements}

The authors would like to thank anonymous reviewers for their valuable comments and suggestions about improving the quality of the manuscript. This work is supported by The National Natural Science Foundation of China under Grant 11071020 and Specialized Research Fund for the Doctoral Program of Higher Education under Grant 20100003110004.

Received: 16 February 2012 Accepted: 2 May 2012 Published: 18 June 2012

\section{References}

1. Kheyfits, A: Dirichlet problem for the Schrödinger operator in a half-space with boundary data of arbitrary growth at infinity. Diff. Integr. Equ. 10, 153-164 (1997)

2. Kheyfits, A: Liouville theorems for generalized harmonic functions. Potential Anal. 16, 93-101 (2002)

3. Reed, M, Simon, B: Methods of Modern Mathematical Physics, vol. 3. Academic Press, London (1970)

4. Rosenblum, G, Solomyak, M, Shubin, M: Spectral Theory of Differential Operators. VINITI, Moscow (1989)

5. Courant, R, Hilbert, D: Methods of Mathematical Physics, 1st English edn. Interscience, New York (1953)

6. Gilbarg, D, Trudinger, NS: Elliptic Partial Differential Equations of Second Order. Springer, Berlin (1977)

7. Muller, C: Spherical Harmonics. Lect. Notes in Math., vol. 17. Springer, Berlin (1966)

8. Simon, B: Schrödinger semigroups. Bull. Am. Math. Soc. 7, 447-526 (1982)

9. Hartman, P: Ordinary Differential Equations. Wiley, New York (1964)

10. Escassut, A, Tutschke, W, Yang, CC: Some Topics on Value Distribution and Differentiability in Complex and P-adic Analysis. Science Press, Beijing (2008)

11. Kheyfits, A: Representation of the analytic functions of infinite order in a half-plane. Izv. Akad. Nauk Armjan SSR Ser. Mat. 6(6), 472-476 (1971)

12. Yoshida, H, Miyamoto, I: Solutions of the Dirichlet problem on a cone with continuous data. J. Math. Soc. Jpn. 50(1), 71-93 (1998)

13. Azarin, VS: Generalization of a theorem of Hayman on subharmonic functions in an $m$-dimensional cone. Trans. Am. Math. Soc. 80(2), 119-138 (1969)

14. Ancona, A: First eigenvalues and comparison of Green's functions for elliptic operators on manifolds or domains. J. Anal. Math. 72, 45-92 (1997) 